\title{
SOME REMARKS ON THE NONLINEAR SCHRÖDINGER EQUATION WITH FRACTIONAL DISSIPATION.
}

\author{
MOHAMAD DARWICH AND LUC MOLINET.
}

\begin{abstract}
We consider the Cauchy problem for the $L^{2}$-critical focussing nonlinear Schrödinger equation with a fractional dissipation. According to the order of the fractional dissipation, we prove the global existence or the existence of finite time blowup dynamics with the log-log blow-up speed for $\|\nabla u(t)\|_{L^{2}}$.
\end{abstract}

\section{INTRODUCTION}

In this paper, we study the blowup and the global existence of solutions for the $L^{2}$-critical nonlinear Schrödinger equation (NLS) with a fractional dissipation term:

$$
\left\{\begin{array}{l}
i u_{t}+\Delta u+|u|^{\frac{4}{d}} u+i a(-\Delta)^{s} u=0,(t, x) \in\left[0, \infty\left[\times \mathbb{R}^{d}, d=1,2,3,4\right.\right. \\
u(0)=u_{0} \in H^{r}\left(\mathbb{R}^{d}\right)
\end{array}\right.
$$

where $a>0$ is the coefficient of friction, $s \geq 0$ and $r \in \mathbb{R}$.

The NLS equation $(a=0)$ arises in various areas of nonlinear optics, plasma physics and fluid mechanics to describe propagation phenomena in dispersive media. To take into account weak dissipation effects, one usually add a damping term as in the linear damped NLS equation (see for instance Fibich [8] ):

$$
i u_{t}+\Delta u+i a u+|u|^{p} u=0, a>0,
$$

or a laplacian term as in the following complex Ginzburg-Landau equation studied in Passota-Sulem-Sulem [25]:

$$
i u_{t}+\Delta u-i a \Delta u+|u|^{p} u=0, a>0,
$$

However, in many cases of practical importance the damping is not described by a local term even in the long-wavelenth limit. In media with dispersion the weak dissipation is, in general, non local (see for instance Ott-Sudan [23]). It is thus quite natural to complete the NLS equation by a non local dissipative term in order to take into account some dissipation phenomena.

In this this paper we complete the $L^{2}$-critical NLS equation (1.2) with a fractional laplacian of order $2 s, s>0$, and study the influence of this term on the blow-up phenomena for this equation.

Recall that the Cauchy problem for the $L^{2}$-critical focussing nonlinear Schrödinger

Key words and phrases. Damped Nonlinear Schrödinger Equation, Blow-up, Global existence. 
equation $(a=0)$ :

$$
\left\{\begin{array}{l}
i u_{t}+\Delta u+|u|^{\frac{4}{d}} u=0 \\
u(0)=u_{0} \in H^{r}\left(\mathbb{R}^{d}\right)
\end{array}\right.
$$

has been studied by a lot of authors (see for instance [13, [4, 3]) and it is known that the problem is locally well-posed in $H^{r}\left(\mathbb{R}^{d}\right)$ for $r \geq 0$ : For any $u_{0} \in H^{r}\left(\mathbb{R}^{d}\right)$, with $r \geq 0$, there exist $T>0$ and a unique solution $u$ of (1.2) with $u(0)=u_{0}$ such that $u \in C\left([[0, T]) ; H^{r}\left(\mathbb{R}^{d}\right)\right)$. Moreover, if $T^{*}$ is the maximal existence time of the solution $u$ in $H^{r}\left(\mathbb{R}^{d}\right)$ then $\lim _{t \rightarrow T^{*}}\|u(t)\|_{L^{2}\left(\mathbb{R}^{d}\right)}=\infty$.

Let us mention that in the case $a>0$ the same results on the Cauchy problem for (1.1) can be established in exactly the same way as in the case $a=0$, since the same Strichartz estimates hold (see for instance [22]).

For $u_{0} \in H^{1}\left(\mathbb{R}^{d}\right)$, a sharp criterion for global existence for (1.2) has been exhibited by Weinstein [29]: Let $Q$ be the unique radial positive solution to

$$
\Delta Q+Q|Q|^{\frac{4}{d}}=Q .
$$

If $\left\|u_{0}\right\|_{L^{2}}<\|Q\|_{L^{2}}$ then the solution of (1.2) is global in $H^{1}$. This follows from the conservation of the energy and the $L^{2}$ norm and the sharp Gagliardo-Nirenberg inequality which ensures that

$$
\forall u \in H^{1}, E(u) \geq \frac{1}{2}\left(\int|\nabla u|^{2}\right)\left(1-\left(\frac{\int|u|^{2}}{\int|Q|^{2}}\right)^{\frac{2}{d}}\right) .
$$

Actually, it was recently proven that any solution of (1.2) emanating from an initial datum $u_{0} \in L^{2}\left(\mathbb{R}^{d}\right)$ with $\left\|u_{0}\right\|_{L^{2}}<\|Q\|_{L^{2}}$ is global and does scatter (cf. [7]).

On the other hand, there exists explicit solutions with $\left\|u_{0}\right\|_{L^{2}}=\|Q\|_{L^{2}}$ that blow up at some time $T>0$ with a $H^{1}$ norm that grows as $\frac{1}{T-t}$.

In the series of papers [17, 27, Merle and Raphael studied the blowup for (1.2) with $\|Q\|_{L^{2}}<\left\|u_{0}\right\|_{L^{2}}<\|Q\|_{L^{2}}+\delta, \delta$ small and proved the existence of the blowup regime corresponding to the log-log law:

$$
\|u(t)\|_{H^{1}\left(\mathbb{R}^{d}\right)} \sim\left(\frac{\log |\log (T-t)|}{T-t}\right)^{\frac{1}{2}} .
$$

Recall that the evolution of (1.2) admits the following conservation laws in the energy space $H^{1}$ :

$$
\begin{aligned}
& L^{2} \text {-norm : } m(u)=\|u\|_{L^{2}}=\left(\int|u(x)|^{2} d x\right)^{1 / 2} . \\
& \text { Energy : } E(u)=\frac{1}{2}\|\nabla u\|_{L^{2}}^{2}-\frac{d}{4+2 d}\|u\|_{L^{\frac{4}{d}+2}}^{\frac{4}{d}+2} .
\end{aligned}
$$

Kinetic momentum : $P(u)=\operatorname{Im}\left(\int \nabla u(x) \bar{u}(x) d x\right.$.

Now, for (1.1) with $a>0$, there does not exist conserved quantities anymore. However, it is easy to prove that if $u$ is a smooth solution of (1.1) on $[0, T[$, then for all $t \in[0, T[$ it holds

$$
\|u(t)\|_{L^{2}}^{2}+a \int_{0}^{t}\left\|(-\Delta)^{\frac{s}{2}} u\right\|_{L^{2}}^{2}=\left\|u_{0}\right\|_{L^{2}}^{2} .
$$




$$
\begin{gathered}
\frac{d}{d t} E(u(t))=-a \int\left((-\Delta)^{\frac{s+1}{2}} u(t)\right)^{2}+a \operatorname{Im} \int(-\Delta)^{s} u(t)|u(t)|^{\frac{4}{d}} \bar{u}(t) . \\
\frac{1}{2} \frac{d}{d t} P(u(t))=a \operatorname{Im} \int(-\Delta)^{s} u(t) \overline{\nabla u}(t) .
\end{gathered}
$$

In [6], the first author studied the case $s=0$. He proved the global existence in $H^{1}$ for $\left\|u_{0}\right\|_{L^{2}} \leq\|Q\|_{L^{2}}$, and showed that the log-log regime is stable by such perturbations (i.e. there exist solutions blows up in finite time with the log-log law).

In [25], Passot, Sulem and Sulem proved that the solutions are global in $H^{1}\left(\mathbb{R}^{2}\right)$ for $s=1$. However, their method does not seem to apply for any other values of $d$.

Our aim in this paper is to establish some results, for $s>0$, on the global existence or the existence of finite time blowup dynamics with the log-log blow-up speed for $\|\nabla u\|_{L^{2}}$.

Let us now state our results:

Theorem 1.1. Let $d=1,2,3,4$ and $0<s<1$ then there exists $\delta_{0}>0$ such that $\forall a>0$ and $\forall \delta \in] 0, \delta_{0}\left[\right.$, there exists $u_{0} \in H^{1}$ with $\left\|u_{0}\right\|_{L^{2}}=\|Q\|_{L^{2}}+\delta$, such that the solution of (1.1) blows up in finite time in the log-log regime.

Theorem 1.2. Let $d=1,2,3,4, s \geq 1$ and $r \geq 0$. Then the Cauchy problem (1.1) is globally well-posed in $H^{r}\left(\mathbb{R}^{d}\right)$.

Theorem 1.3. Let $0<s<1$ and $a>0$.

(1) There exists a real number $0<\gamma=\gamma(d) \leq\|Q\|_{L^{2}}$ such that for any initial datum $u_{0} \in H^{1}\left(\mathbb{R}^{d}\right)$ with $\left\|u_{0}\right\|_{L^{2}}<\gamma$, the emanating solution $u$ is global in $H^{1}$ with an energy that is non increasing.

(2) There does not exists any intial datum $u_{0}$, with $\left\|u_{0}\right\|_{L^{2}} \leq\|Q\|_{L^{2}}$, such that the solution $u$ of (1.1) blows up at finite time $T^{*}$ and satisfies

$$
\frac{1}{\left(T^{*}-t\right)^{\alpha}} \lesssim\|\nabla u(t)\|_{L^{2}\left(\mathbb{R}^{d}\right)} \lesssim \frac{1}{\left(T^{*}-t\right)^{\beta}}, \quad \forall 0<T-t \ll 1,
$$

for some pair $(\alpha, \beta)$ satisfying $0<\beta<\frac{1}{2 s}$ and $\beta(1+s)-1 / 2<\alpha \leq \beta$.

Remark 1.1. Note that, assertion (2) of Theorem 1.3 ensures that we do not have any blowup in the log-log regime for any $0<s<1$ and in the regime $\frac{1}{t}$ for any $0<s<\frac{1}{2}$, for initial data with critical or subcritical mass.

Acknowledgments : The first author thanks the L.M.P.T. for his kind hospitality during the development of this work. Moreover, he would like to thank AUF for supporting this project.

\section{LocAl AND GLOBAL EXISTENCE RESUlts}

In this section, we prove Theorem 1.2 and part (1) of Theorem 1.3 , Theorem 1.2 will follow from an a priori estimate on the critical Strichartz norm whereas part (1) of Theorem 1.3 follows from a monotonicity of the energy. 
2.1. Local existence result. Recall that the main tools to prove the local existence results for (1.2) are the Strichartz estimates for the associated linear propagator $e^{i \Delta t}$. These Strichartz estimates reads

$$
\left\|e^{i \Delta t} \phi\right\|_{L_{t}^{q} L^{r}\left(\mathbb{R}^{d}\right)} \lesssim\|\phi\|_{L^{2}\left(\mathbb{R}^{d}\right)}
$$

for any pair $(q, r)$ satisfying $\frac{2}{q}+\frac{d}{r}=\frac{d}{2}$ and $2<q \leq \infty$. Such ordered pair is called an admissible pair .

For $a \geq 0$ and $s \geq 0$ we denote by $S_{a, s}$ the linear semi-group associated with (1.1), i.e. $S_{a, s}(t)=e^{i \Delta t-a(-\Delta)^{s} t}$. The following lemma ensures that the linear semi-group $S_{a, s}$ enjoys the same Strichartz estimates as $e^{i \Delta t}$.

Lemma 2.1. Let $\phi \in L^{2}\left(\mathbb{R}^{d}\right)$. Then for every admissible pair $(q, r)$ it holds

$$
\left\|S_{a, s}(\cdot) \phi\right\|_{L_{t>0}^{q} L^{r}\left(\mathbb{R}^{d}\right)} \lesssim\|\phi\|_{L^{2}\left(\mathbb{R}^{d}\right)} .
$$

Proof. Setting, for any $t \geq 0, G_{a, s}(t, x)=\int e^{-i x \xi} e^{-a t|\xi|^{2 s}} d \xi$, it holds

$$
S_{a, s}(t) \varphi=G_{a, s}(t, \cdot) * e^{i t \Delta} \varphi, \quad \forall t \geq 0 .
$$

Noticing that $\left\|G_{a, s}(t, .)\right\|_{L^{1}}=\left\|G_{1, s}(1, .)\right\|_{L^{1}}$ and that, according to Lemma 2.1 in $[22], G_{1, s}(1,.) \in L^{1}\left(\mathbb{R}^{d}\right)$, we get

$\left.\| S_{a, s}(t) \phi\right)\left\|_{L_{t>0}^{p} L^{q}\left(\mathbb{R}^{d}\right)}=\right\| G_{a, s}(t,). * e^{i t \Delta}(\phi)\left\|_{L_{t>0}^{p} L^{q}\left(\mathbb{R}^{d}\right)} \lesssim\right\| e^{i t \Delta} \phi\left\|_{L_{t}^{p} L^{q}\left(\mathbb{R}^{d}\right)} \lesssim\right\| \phi \|_{L^{2}}$.

With Lemma 2.1 in hand, it is not too hard to check that the local existence results for equation (1.2) (see for instance [4] and [3]) also holds for (1.1) with $a \geq 0$ and $s \geq 0$. More precisely, we have the following statement:

Proposition 2.1. Let $a \geq 0, s \geq 0$ and $u_{0} \in H^{s}\left(\mathbb{R}^{d}\right)$ with $d \in\{1,2,3,4\}$. There exists $T>0$ and a unique solution $u \in C\left([0, T] ; H^{s}\right) \cap L_{T}^{\frac{4}{d}+2} L^{\frac{4}{d}+2}$ to (1.1) emanating from $u_{0}$. In addition, there exists a neighborhood $\mathcal{V}_{u_{0}}$ of $u_{0}$ in $H^{s}$ such that the associated solution map is continuous from $\mathcal{V}_{u_{0}}$ into $C\left([0, T] ; H^{s}\right) \cap L_{T}^{\frac{4}{d}+2} L^{\frac{4}{d}+2}$.

Finally, let $T^{*}$ be the maximal time of existence of the solution $u$ in $H^{s}\left(\mathbb{R}^{d}\right)$, then

$$
T^{*}<\infty \Longrightarrow\|u\|_{L_{T}^{\frac{4}{d}+2} L^{\frac{4}{d}+2}}=+\infty .
$$

2.2. Proof of Theorem 1.2. Let $u \in C\left([0, T] ; L^{2}\left(\mathbb{R}^{d}\right)\right.$ be the solution emanating from some initial datum $u_{0} \in L^{2}\left(\mathbb{R}^{d}\right)$. We have the following a priori estimates:

Lemma 2.2. Let $u \in C\left([0, T] ; L^{2}\left(\mathbb{R}^{d}\right)\right)$ be the solution of (1.1) emanating from $u_{0} \in L^{2}\left(\mathbb{R}^{d}\right)$. Then

$$
\|u\|_{L_{T}^{\infty} L^{2}} \leq\left\|u_{0}\right\|_{L^{2}} \text { and }\left\|(-\Delta)^{\frac{s}{2}} u\right\|_{L_{T}^{2} L^{2}} \leq \frac{1}{\sqrt{2 a}}\left\|u_{0}\right\|_{L^{2}} .
$$


Proof. Assume first that $u_{0} \in H^{\infty}\left(\mathbb{R}^{d}\right)$. Then (1.6) ensures that the mass is decreasing as soon as $u$ is not the null solution and (1.6) leads to

$$
\int_{0}^{T}\left\|(-\Delta)^{\frac{s}{2}} u(t)\right\|_{L^{2}}^{2} d t=-\frac{1}{2 a}\left(\|u(T)\|_{L^{2}}^{2}-\left\|u_{0}\right\|_{L^{2}}^{2}\right) \leq \frac{1}{2 a}\left\|u_{0}\right\|_{L^{2}}^{2} .
$$

This proves (2.2) for smooth solutions. The result for $u_{0} \in L^{2}\left(\mathbb{R}^{d}\right)$ follows by approximating $u_{0}$ in $L^{2}$ by a smooth sequence $\left(u_{0, n}\right) \subset H^{\infty}\left(\mathbb{R}^{d}\right)$.

Note that the first estimate in (2.2) implies that $\|u\|_{L_{T}^{2} L^{2}} \leq T^{1 / 2}\left\|u_{0}\right\|_{L^{2}}$ and thus by interpolation:

$$
\|\nabla u\|_{L_{T}^{2} L^{2}} \lesssim\left\|(-\Delta)^{\frac{s}{2}} u\right\|_{L_{T}^{2} L^{2}}^{\frac{1}{s}}\|u\|_{L^{2} T L^{2}}^{1-\frac{1}{s}} \lesssim T^{\frac{1}{2}\left(1-\frac{1}{s}\right)}
$$

Interpolating now between (2.3) and the first estimate of (2.2) we get

$$
\|u\|_{L_{T}^{\frac{4}{d}+2} H^{\frac{2 d}{4+2 d}}} \lesssim T^{\frac{1}{2}\left(1-\frac{1}{s}\right)}
$$

and the embedding $H^{\frac{2 d}{4+2 d}}\left(\mathbb{R}^{d}\right) \hookrightarrow L^{\frac{4}{d}+2}\left(\mathbb{R}^{d}\right)$ ensures that

$$
\|u\|_{L_{T}^{\frac{4}{d}+2} L^{\frac{4}{d}+2}} \lesssim\|u\|_{L_{T}^{\frac{4}{d}+2} H^{\frac{2 d}{4+2 d}}} \lesssim T^{\frac{1}{2}\left(1-\frac{1}{s}\right)} .
$$

Denoting by $T^{*}$ the maximal time of existence of $u$ in $L^{2}\left(\mathbb{R}^{d}\right)$ and letting $T$ tends to $T^{*}$, this contradicts (2.1) whenever $T^{*}$ is finite. This proves that the solutions are global in $H^{r}\left(\mathbb{R}^{d}\right)$.

2.3. Proof of Assertion 1 of Theorem 1.3. Note that the global existence for any $u_{0} \in L^{2}\left(\mathbb{R}^{d}\right)$ with $\left\|u_{0}\right\|_{L^{2}}$ small enough can be proven, as for the critical NLS equation, directly by a fixed point argument thanks to Lemma 2.1. This ensures the global existence in $H^{r}\left(\mathbb{R}^{d}\right), r \geq 0$, under the same smallness condition on $\left\|u_{0}\right\|_{L^{2}}$. We will not invoke this fact here and we will directly prove Assertion 1 of Theorem 1.3 by combining (1.4) and a monotony result on $t \mapsto E(u(t))$. To do this, we will work with smooth solutions and then get the result for $H^{1}$-solutions by continuity with respect initial data.

So, let $u \in C\left([0, T] ; H^{\infty}\left(\mathbb{R}^{d}\right)\right)$ be a solution to (1.1) emanating from $u_{0} \in$ $H^{\infty}\left(\mathbb{R}^{d}\right)$. Then it holds

$$
\frac{d}{d t} E\left(u(t)=-a \int\left|(-\Delta)^{\frac{s+1}{2}} u(t)\right|^{2}+a \operatorname{Im} \int(-\Delta)^{s} u(t)|u(t)|^{\frac{4}{d}} \overline{u(t)}\right.
$$

and Hölder inequalities in physical space and in Fourier space lead to

$$
\left.\left|\int(-\Delta)^{s} u\right| u\right|^{\frac{4}{d}} \bar{u} \mid \leq\left\|(-\Delta)^{s} u\right\|_{L^{2}}\|u\|_{L^{\frac{8}{d}+2}}^{\frac{4}{d}+1}
$$

with

$$
\left\|(-\Delta)^{s} u\right\|_{L^{2}} \leq\left\|(-\Delta)^{\frac{s+1}{2}} u\right\|_{L^{2}}^{\frac{2 s}{s+1}}\|u\|_{L^{2}}^{\frac{1-s}{1+s}} .
$$

Let us recall the following Gagliardo-Nirenberg inequality (1)

$$
\|u\|_{L^{\frac{8}{d}+2}}^{\frac{8}{d}+2} \leq C_{d}^{\frac{8}{d}+2}\|\nabla u\|_{L^{2}}^{4}\|u\|_{L^{2}}^{\frac{8}{d}-2} .
$$

\footnotetext{
${ }^{(1)}$ It is proven in 29 that the constant $C_{d}$ is related for $d=1,2,3$ to the $L^{2}$-norm of the ground state solution of $2 \Delta \psi-\left(\frac{4}{d}-1\right) \psi+\psi^{\frac{8}{d}+1}=0$.
} 
This estimate together with Cauchy-Schwarz inequality (in Fourier space)

$$
\|\nabla u\|_{L^{2}}^{2} \leq\left\|(-\Delta)^{\frac{s+1}{2}} u\right\|_{L^{2}}^{\frac{2}{s+1}}\|u\|_{L^{2}}^{\frac{2 s}{s+1}}
$$

lead to

$$
\|u\|_{L^{\frac{8}{d}}+2}^{\frac{4}{d}+1} \leq C^{\frac{4}{d}+1}\left\|(-\Delta)^{\frac{s+1}{2}} u\right\|_{L^{2}}^{\frac{2}{s+1}}\|u\|_{L^{2}}^{\frac{2 s}{s+1}}\|u\|_{L^{2}}^{\frac{4-d}{d}} .
$$

Combining the above estimates we eventually obtain

$$
\frac{d}{d t} E(u(t)) \leq a\left\|(-\Delta)^{\frac{s+1}{2}} u\right\|_{L^{2}}^{2}\left(C^{\frac{4}{d}+1}\|u\|_{L^{2}}^{4 / d}-1\right)
$$

which together with (2.2) implies that $E(u(t))$ is not increasing for $\left\|u_{0}\right\|_{L^{2}} \leq$ $C^{1+\frac{d}{4}}$.

\section{Proof of Assertion 2 of Theorem 1.3}

Special solutions play a fundamental role for the description of the dynamics of (NLS). They are the solitary waves of the form $u(t, x)=\exp (i t) Q(x)$, where $Q$ the unique positive radial solution to

$$
\Delta Q+Q|Q|^{\frac{4}{d}}=Q
$$

The pseudo-conformal transformation applied to the "stationary" solution $e^{i t} Q(x)$ yields an explicit solution for (NLS)

$$
S(t, x)=\frac{1}{|t|^{\frac{d}{2}}} Q\left(\frac{x}{t}\right) e^{-i \frac{|x|^{2}}{4 t}+\frac{i}{t}}
$$

which blows up at $T^{*}=0$.

Note that

$$
\|S(t)\|_{L^{2}}=\|Q\|_{L^{2}} \text { and }\|\nabla S(t)\|_{L^{2}} \sim \frac{1}{t}
$$

It turns out that $S(t)$ is the unique minimal mass blow-up solution in $H^{1}$ up to the symmetries of the equation ( see [16]).

A known lower bound ( see [21]) on the blow-up rate for (NLS) is

$$
\|\nabla u(t)\|_{L^{2}} \geq \frac{C\left(u_{0}\right)}{\sqrt{T-t}} .
$$

Note that this blow-up rate is strictly lower than the one of $S(t)$ given by (3.2) and of the log-log law given by (1.5).

To prove assertion 2 of Theorem [1.3, we will need the following result see [12]) :

Theorem 3.1. Let $\left(v_{n}\right)_{n}$ be a bounded family of $H^{1}\left(\mathbb{R}^{d}\right)$, such that:

$$
\limsup _{n \rightarrow+\infty}\left\|\nabla v_{n}\right\|_{L^{2}\left(\mathbb{R}^{d}\right)} \leq M \quad \text { and } \quad \limsup _{n \rightarrow+\infty}\left\|v_{n}\right\|_{L^{\frac{4}{d}+2}} \geq m \text {. }
$$

Then, there exists $\left(x_{n}\right)_{n} \subset \mathbb{R}^{d}$ such that:

$$
v_{n}\left(\cdot+x_{n}\right) \rightarrow V \quad \text { weakly },
$$

with $\|V\|_{L^{2}\left(\mathbb{R}^{d}\right)} \geq\left(\frac{d}{d+4}\right)^{\frac{d}{4}} \frac{m^{\frac{d}{2}+1}+1}{M^{\frac{d}{2}}}\|Q\|_{L^{2}\left(\mathbb{R}^{d}\right)}$. 
Suppose that there exist an initial data $u_{0}$ with $\left\|u_{0}\right\|_{L^{2}} \leq\|Q\|_{L^{2}}$, such that the corresponding solution $u(t)$ blows up at time $T>0$ with the following behavior:

$$
\frac{1}{(T-t)^{\alpha}} \lesssim\|\nabla u(t)\|_{L^{2}\left(\mathbb{R}^{d}\right)} \lesssim \frac{1}{(T-t)^{\beta}}, \quad \forall t \in[0, T[,
$$

where $\beta>0$ and $\alpha \geq \beta$ satisfies $\alpha>\beta(1+s)-1 / 2)$.

Recalling that

$$
E(u(t))=E\left(u_{0}\right)-a \int_{0}^{t} K(u(\tau)) d \tau, \quad t \in[0, T[,
$$

with $K(u(t))=\int\left|(-\Delta)^{\frac{s+1}{2}} u\right|^{2}-\operatorname{Im} \int(-\Delta)^{s} u|u|^{\frac{4}{d}} \bar{u}$, we obtain that

$E(u(t)) \lesssim E\left(u_{0}\right)+\left.\left|\int_{0}^{t}(-\Delta)^{s}(u)\right| u\right|^{\frac{4}{d}} \bar{u} d x \mid \lesssim E\left(u_{0}\right)+\int_{0}^{t}\left\|(-\Delta)^{s}(u)\right\|_{L^{2}}\|u\|_{L^{\frac{8}{d}+2}}^{\frac{4}{d}+1}$

This last estimate together with

$\|u\|_{L^{\frac{8}{d}+2}}^{\frac{4}{d}+1} \lesssim\|\nabla u\|_{L^{2}}^{2}\left\|u_{0}\right\|_{L^{2}}^{\frac{4-d}{d}} \quad$ and $\quad\left\|(-\Delta)^{s}(u)\right\|_{L^{2}} \leq\|\nabla u\|_{L^{2}}^{2 s}\|u\|_{L^{2}}^{1-2 s} \lesssim\|\nabla u\|_{L^{2}}^{2 s}$ yield

$$
E(u(t)) \lesssim E\left(u_{0}\right)+\int_{0}^{t}\|\nabla u\|_{L^{2}}^{2+2 s}(\tau) d \tau .
$$

Note that assumption (3.5) ensures that

$$
0 \leq \frac{\int_{0}^{t}\|\nabla u(\tau)\|_{L^{2}}^{2+2 s} d \tau}{\|\nabla u(t)\|_{L^{2}\left(\mathbb{R}^{d}\right)}^{2}} \lesssim(T-t)^{-2 \beta(1+s)+1+2 \alpha} \rightarrow 0 \text { as } t \nearrow T
$$

Now, let

$$
\rho(t)=\frac{\|\nabla Q\|_{L^{2}\left(\mathbb{R}^{d}\right)}}{\|\nabla u(t)\|_{L^{2}\left(\mathbb{R}^{d}\right)}} \quad \text { and } \quad v(t, x)=\rho^{\frac{d}{2}} u(t, \rho x)
$$

and let $\left(t_{k}\right)_{k}$ be a sequence of positive times such that $t_{k} \nearrow T$. We set $\rho_{k}=\rho\left(t_{k}\right)$ and $v_{k}=v\left(t_{k},.\right)$. The family $\left(v_{k}\right)_{k}$ satisfies

$\left\|v_{k}\right\|_{L^{2}\left(\mathbb{R}^{d}\right)}=\left\|u\left(t_{k}, \cdot\right)\right\|_{L^{2}\left(\mathbb{R}^{d}\right)}<\left\|u_{0}\right\|_{L^{2}\left(\mathbb{R}^{d}\right)} \leq\|Q\|_{L^{2}\left(\mathbb{R}^{d}\right)} \quad$ and $\quad\left\|\nabla v_{k}\right\|_{L^{2}\left(\mathbb{R}^{d}\right)}=\|\nabla Q\|_{L^{2}\left(\mathbb{R}^{d}\right)}$.

The above estimate on $\left\|v_{k}\right\|_{L^{2}}$ and (3.7) lead to

$0<\frac{1}{2}\left(\int\left|\nabla v_{k}\right|^{2}\right)\left(1-\left(\frac{\int\left|v_{k}\right|^{2}}{\int|Q|^{2}}\right)^{2}\right) \leq E\left(v_{k}\right)=\rho_{k}^{2} E(u(t)) \lesssim \rho_{k}^{2} E\left(u_{0}\right)+\rho_{k}^{2} \int_{0}^{t_{k}}\|\nabla u(\tau)\|_{L^{2}}^{2+2 s} d \tau$

which, together with (3.8), ensures that $\lim _{k \rightarrow+\infty} E\left(v_{k}\right)=0$. This forces

$$
\left\|v_{k}\right\|_{L^{\frac{4}{d}+2}}^{\frac{4}{d}+2} \rightarrow \frac{d+2}{d}\left\|\nabla v_{k}\right\|_{L^{2}\left(\mathbb{R}^{d}\right)}^{2}=\frac{d+2}{d}\|\nabla Q\|_{L^{2}\left(\mathbb{R}^{d}\right)}^{2}
$$

and thus the family $\left(v_{k}\right)_{k}$ satisfies the hypotheses of Theorem 3.1 with

$$
m^{\frac{4}{d}+2}=\frac{d+2}{d}\|\nabla Q\|_{L^{2}\left(\mathbb{R}^{d}\right)}^{2} \quad \text { and } \quad M=\|\nabla Q\|_{L^{2}\left(\mathbb{R}^{d}\right)} .
$$

Hence, there exists a family $\left(x_{k}\right)_{k} \subset \mathbb{R}$ and a profile $V \in H^{1}(\mathbb{R})$ with 
$\|V\|_{L^{2}\left(\mathbb{R}^{d}\right)} \geq\|Q\|_{L^{2}\left(\mathbb{R}^{d}\right)}$, such that,

$$
\rho_{k}^{\frac{d}{2}} u\left(t_{k}, \rho_{k} \cdot+x_{k}\right) \rightarrow V \in H^{1} \quad \text { weakly. }
$$

Using (3.10),,$\forall A \geq 0$

$$
\liminf _{n \rightarrow+\infty} \int_{B(0, A)} \rho_{n}^{d}\left|u\left(t_{n}, \rho_{n} x+x_{n}\right)\right|^{2} d x \geq \int_{B(0, A)}|V|^{2} d x
$$

But, since $\lim _{n \rightarrow+\infty} \rho_{n}=0, \quad \rho_{n} A<1$ for $n$ large enough and thus

$$
\liminf _{n \rightarrow+\infty} \sup _{y \in \mathbb{R}} \int_{|x-y| \leq 1}\left|u\left(t_{n}, x\right)\right|^{2} d x \geq \liminf _{n \rightarrow+\infty} \int_{\left|x-x_{n}\right| \leq \rho_{n} A}\left|u\left(t_{n}, x\right)\right|^{2} d x \geq \int_{|x| \leq A}|V|^{2} d x .
$$

Since this it is true for all $A>0$ we obtain that

$$
\left\|u_{0}\right\|_{L^{2}}^{2}>\liminf _{n \rightarrow+\infty} \sup _{y \in \mathbb{R}} \int_{|x-y| \leq 1}\left|u\left(t_{n}, x\right)\right|^{2} d x \geq\|Q\|_{L^{2}}^{2}
$$

which contradicts the assumption $\left\|u_{0}\right\|_{L^{2}} \leq\|Q\|_{L^{2}}$ and the desired result is proven.

\section{Blow up SOlution.}

In this section, we prove the existence of the explosive solutions in the case $0<s<1$.

Theorem 4.1. Let $0<s<1$. There exist a set of initial data $\Omega$ in $H^{1}$, such that for any $0<a<a_{0}$ with $a_{0}=a_{0}(s)$ small enough, the emanating solution $u(t)$ to (1.1) blows up in finite time in the log-log regime.

The set of initial data $\Omega$ is the set described in [17, in order to initialize the log-log regime. It is open in $H^{1}$. Using the continuity with regard to the initial data and the parameters, we easily obtain the following corollary:

Corollary 4.1. Let $0<s<1$ and $u_{0} \in H^{1}$ be an initial data such that the corresponding solution $u(t)$ of (1.2) blows up in the loglog regime. There exist $\beta_{0}>0$ and $a_{0}>0$ such that if $v_{0}=u_{0}+h_{0},\left\|h_{0}\right\|_{H^{1}} \leq \beta_{0}$ and $a \leq a_{0}$, the solution $v(t)$ for (1.1) with the initial data $v_{0}$ blows up in finite time.

Now to prove Theorem 4.1. we look for a solution of (1.1) such that for $t$ close enough to blowup time, we shall have the following decomposition:

$$
u(t, x)=\frac{1}{\lambda^{\frac{d}{2}}(t)}\left(Q_{b(t)}+\epsilon\right)\left(t, \frac{x-x(t)}{\lambda(t)}\right) e^{i \gamma(t)},
$$

for some geometrical parameters $(b(t), \lambda(t), x(t), \gamma(t)) \in(0, \infty) \times(0, \infty) \times$ $\mathbb{R}^{d} \times \mathbb{R}$, here $\lambda(t) \sim \frac{1}{\|\nabla u(t)\|_{L^{2}}}$, and the profiles $Q_{b}$ are suitable deformations of $Q$ related to some extra degeneracy of the problem.

Note that we will abbreviated our proof because it is very close to the case of $s=0$ ( see Darwich [6]). Actually, as noticed in [26, we only need to prove that in the log-log regime the $L^{2}$ norm does not grow, and the growth of the energy ( resp the momentum) is below $\frac{1}{\lambda(t)^{2}}\left(\operatorname{resp} \frac{1}{\lambda(t)}\right)$. In this paper, 
we will prove that in the log-log regime, the growths of the energy and the momentum are bounded by:

$$
E(u(t)) \lesssim \log (\lambda(t)) \lambda(t)^{-2 s}, P(u(t)) \lesssim \log (\lambda(t)) \lambda(t)^{\frac{-2 s}{s+1}} .
$$

Let us recall that a fonction $\mathrm{u}:[0, T] \longmapsto H^{1}$ follows the log-log regime if the following uniform controls on the decomposition (4.1) hold on $[0, T]$ :

- Control of $b(t)$

$$
b(t)>0, b(t)<10 b(0) .
$$

- Control of $\lambda$ :

$$
\lambda(t) \leq e^{-e^{\frac{\pi}{100 b(t)}}}
$$

and the monotonicity of $\lambda$ :

$$
\lambda\left(t_{2}\right) \leq \frac{3}{2} \lambda\left(t_{1}\right), \forall 0 \leq t_{1} \leq t_{2} \leq T .
$$

Let $k_{0} \leq k_{+}$be integers and $T^{+} \in[0, T]$ such that

$$
\frac{1}{2^{k_{0}}} \leq \lambda(0) \leq \frac{1}{2^{k_{0}-1}}, \frac{1}{2^{k_{+}}} \leq \lambda\left(T^{+}\right) \leq \frac{1}{2^{k_{+}-1}}
$$

and for $k_{0} \leq k \leq k_{+}$, let $t_{k}$ be a time such that

$$
\lambda\left(t_{k}\right)=\frac{1}{2^{k}}
$$

then we assume the control of the doubling time interval:

$$
t_{k+1}-t_{k} \leq k \lambda^{2}\left(t_{k}\right)
$$

- control of the excess of mass:

$$
\int_{\mathbb{R}^{d}}|\nabla \epsilon(t)|^{2}+\int_{\mathbb{R}^{d}}|\epsilon(t)|^{2} e^{-|y|} \leq \Gamma_{b(t)}^{\frac{1}{4}}, \text { where } \Gamma_{b} \sim e^{-\frac{\pi}{b}} .
$$

The main point is to establish that (4.2)-(4.8) determine a trapping region for the flow. Actually, after the decomposition (4.1) of $u$, the log-log regime corresponds to the following asymptotic controls

$$
b_{s} \sim C e^{-\frac{c}{b}},-\frac{\lambda_{s}}{\lambda} \sim b
$$

and

$$
\int_{\mathbb{R}^{d}}|\nabla \epsilon|^{2} \lesssim e^{-\frac{c}{b}}
$$

where we have introduced the rescaled time $\frac{d s}{d t}=\frac{1}{\lambda^{2}}$.

In fact, (4.10) is partly a consequence of the preliminary estimate:

$$
\int_{\mathbb{R}^{d}}|\nabla \epsilon|^{2} \lesssim e^{-\frac{c}{b}}+\lambda^{2}(t) E(t)
$$

One then observes that in the log-log regime, the integration of the laws (4.9) yields

$$
\lambda \sim e^{-e^{\frac{c}{b}}} \ll e^{-\frac{c}{b}}, b(t) \rightarrow 0, t \rightarrow T .
$$

Hence, the term involving the conserved Hamiltonian is asymptotically negligible with respect to the leading order term $e^{-\frac{c}{b}}$ which drives the decay (4.11) of $b$. This was a central observation made by Planchon and Raphael in [26]. In fact, any growth of the Hamiltonian algebraically below $\frac{1}{\lambda^{2}(t)}$ 
would be enough. In this paper, we will prove that in the log-log regime, the growth of the energy is estimated by:

$$
E(u(t)) \lesssim(\log (\lambda(t))) \lambda^{-2 s}(t) .
$$

It then follows from (4.11) that:

$$
\int_{\mathbb{R}^{d}}|\nabla \epsilon|^{2} \lesssim e^{-\frac{c}{b}}
$$

An important feature of this estimate of $H^{1}$ flavor is that it relies on a flux computation in $L^{2}$. This allows one to recover the asymptotic laws for the geometrical parameters (4.9) and to close the bootstrap estimates of the $\log$-log regime.

Remark 4.1. Actually, one also needs the bound on the momentum to control the geometrical parameters (see Lemma 7.2 in [6] ).

4.1. Control of the energy and the kinetic momentum. Let us recall that we say that an ordered pair $(q, r)$ is admissible whenever $\frac{2}{q}+\frac{d}{r}=\frac{d}{2}$ and $2<q \leq \infty$. We define the Strichartz norm of functions $u:[0, T] \times \mathbb{R}^{d} \longmapsto \mathbb{C}$ by:

$$
\|u\|_{S^{0}\left([0, T] \times \mathbb{R}^{d}\right)}=\sup _{(q, r) \text { admissible }}\|u\|_{L_{t}^{q} L_{x}^{r}\left([0, T] \times \mathbb{R}^{d}\right)}
$$

and

$$
\|u\|_{S^{1}\left([0, T] \times \mathbb{R}^{d}\right)}=\sup _{(q, r) \text { admissible }}\|\nabla u\|_{L_{t}^{q} L_{x}^{r}\left([0, T] \times \mathbb{R}^{d}\right)}
$$

We will sometimes abbreviate $S^{i}\left([0, T] \times \mathbb{R}^{2}\right)$ with $S_{T}^{i}$ or $S^{i}[0, T], i=1,2$. Now we will derive an estimate on the energy, to check that it remains small with respect to $\lambda^{-2}$ :

Proposition 4.1. Assuming that (4.3)-(4.8) hold, then the energy and kinetic momentum are controlled on $\left[0, T^{+}\right]$by:

$$
\begin{aligned}
& |E(u(t))| \lesssim(\log (\lambda(t))) \lambda^{-2 s}(t), \\
& |P(u(t))| \lesssim(\log (\lambda(t))) \lambda^{\frac{-2 s}{s+1}}(t) .
\end{aligned}
$$

To prove Proposition 4.1, we will need the two following lemmas.

Lemma 4.1. Let $u \in C\left([0, T] ; H^{1}\left(\mathbb{R}^{d}\right)\right)$ be a solution of $[1.1)$. Then we have the following estimation:

$$
\|\nabla u\|_{L_{T}^{\infty} L_{x}^{2}}+\left\|(\Delta)^{\frac{s+1}{2}} u\right\|_{L_{T}^{2} L_{x}^{2}} \lesssim\left\|\nabla u_{0}\right\|_{L_{x}^{2}}+\left\||u|^{\frac{4}{d}} \nabla u\right\|_{L_{T}^{1} L_{x}^{2}}
$$

Proof. Multiply Equation 1.1 by $\overline{\Delta u}$, integrate and take the imaginary part, to obtain :

$$
\frac{1}{2} \frac{d}{d t}\|\nabla u\|^{2}+a \int\left|(-\Delta)^{\frac{s+1}{2}} u\right|^{2} \leq\left.\left|\int\right| u\right|^{\frac{4}{d}} u \Delta u|=| \int \nabla\left(|u|^{\frac{4}{d}} u\right) \nabla u \mid
$$

By integrating in time, we get

$$
\frac{1}{2}\|\nabla u\|_{L_{T}^{\infty} L^{2}}^{2}+a\left\|(-\Delta)^{\frac{s+1}{2}} u\right\|_{L_{T}^{2} L_{x}^{2}}^{2} \leq \frac{1}{2}\left\|\nabla u_{0}\right\|_{L^{2}}^{2}+\left\|\nabla\left(|u|^{\frac{4}{d}} u\right)\right\|_{L_{T}^{1} L_{x}^{2}}\|\nabla u\|_{L_{T}^{\infty} L^{2}}
$$


Dividing by $\sqrt{\frac{1}{2}\|\nabla u\|_{L_{T}^{\infty} L^{2}}^{2}+a\left\|(-\Delta)^{\frac{s+1}{2}} u\right\|_{L_{T}^{2} L_{x}^{2}}^{2}}$ we obtain:

$$
\|\nabla u\|_{L_{T}^{\infty} L_{x}^{2}}+\left\|(\Delta)^{\frac{s+1}{2}} u\right\|_{L_{T}^{2} L_{x}^{2}} \lesssim\left\|\nabla u_{0}\right\|_{L^{2}}+\left\||u|^{\frac{4}{d}} \nabla u\right\|_{L_{T}^{1} L_{x}^{2}}
$$

Lemma 4.2. There exists a real number $0<\alpha \ll 1$ such that the following holds: Let $u \in C\left([0, T] ; H^{1}\left(\mathbb{R}^{d}\right)\right)$ be the solution of (1.1) emanating from $u_{0} \in H^{1}$. For a fixed $\left.t \in\right] 0, T\left[\right.$ we set $\Delta t=\alpha\left\|u_{0}\right\|_{L^{2}}^{\frac{d-4}{d}}\|u(t)\|_{H^{1}}^{-2}$. Then $u \in C\left([t, t+\Delta t] ; H^{1}\left(\mathbb{R}^{d}\right)\right)$ and we have the following controls

and

$$
\|u\|_{S^{0}[t, t+\Delta t]} \leq 2\left\|u_{0}\right\|_{L^{2}}
$$

$$
\|u\|_{S^{1}[t, t+\Delta t]}+\left\|(-\Delta)^{\frac{s+1}{2}} u\right\|_{L^{2}(] t, t+\Delta t[) L_{x}^{2}} \leq 2\|u(t)\|_{H^{1}\left(\mathbb{R}^{d}\right)}
$$

Proof. We first assume that $\Delta t>0$ is such that $t+\Delta t<T$. Then, according to Lemma 2.1, it holds

$$
\left\|\int_{0}^{t} S_{a, s}(t-\tau)|u(\tau)|^{\frac{4}{d}} u(\tau) d \tau\right\|_{S^{1}[t, t+\Delta t]} \lesssim\left\||u|^{\frac{4}{d}} \nabla u\right\|_{L^{1}(] t, t+\Delta t[) L_{x}^{2}} .
$$

Using the Hölder inequality we obtain:

$$
\left(\int|u|^{\frac{8}{d}}|\nabla u|^{2}\right)^{\frac{1}{2}} \leq\left(\int|u|^{\frac{2(4+d)}{d}}\right)^{\frac{2}{4+d}}\left(\int|\nabla u|^{\frac{2(4+d)}{d}}\right)^{\frac{d}{2(4+d)}} .
$$

Integrating in time and applying again Hölder inequality we get:

$$
\begin{aligned}
\left\||u|^{\frac{4}{d}} \nabla u\right\|_{\left.L^{1}\right] t, t+\Delta t[) L^{2}\left(\mathbb{R}^{d}\right)} & \leq\left(\int\left(\int|u|^{\frac{2(4+d)}{d}} d x\right)^{\frac{2}{4+d} \frac{4+d}{4}} d t\right)^{\frac{4}{4+d}} \\
& \times\left(\int\left(\int|\nabla u|^{\frac{2(4+d)}{d}} d x\right)^{\frac{d}{2(4+d)} \frac{4+d}{d}} d t\right)^{\frac{d}{4+d}} .
\end{aligned}
$$

Thus:

$$
\left\||u|^{\frac{4}{d}} \nabla u\right\|_{L^{1}(] t, t+\Delta t[) L^{2}\left(\mathbb{R}^{d}\right)} \leq\|u\|_{L^{\frac{4+d}{d}}(] t, t+\Delta t[) L^{\frac{4+2 d}{d}}\left(\mathbb{R}^{d}\right)}\|\nabla u\|_{L^{\frac{4+d}{d}}(] t, t+\Delta t[) L^{\frac{8+2 d}{d}}\left(\mathbb{R}^{d}\right)} .
$$

But $\left(\frac{4+d}{d}, \frac{8+2 d}{d}\right)$ is admissible, thus we have:

$$
\left\||u|^{\frac{4}{d}} \nabla u\right\|_{L^{1}([t, t+\Delta t]) L^{2}\left(\mathbb{R}^{d}\right)} \leq\|u\|_{L^{\frac{4+d}{d}}([t, t+\Delta t]) L^{\frac{8+2 d}{d}}\left(\mathbb{R}^{d}\right)}^{\frac{4}{d}}\|u\|_{S^{1}[t, t+\Delta t]} .
$$

By Sobolev inequalities we have:

$$
\begin{aligned}
\|u\|_{L}{ }^{\frac{4+d}{d}[t, t+\Delta t]_{L} \frac{8+2 d}{d}\left(\mathbb{R}^{d}\right)} & \lesssim\|u\|_{L^{\frac{4+d}{d}}([t, t+\Delta t]) H^{\frac{2 d}{d+4}}\left(\mathbb{R}^{d}\right)} \\
& \leq(\Delta t)^{\frac{d}{d+4}}\|u\|_{L^{\infty}([t, t+\Delta t]) H^{\frac{2 d}{d+4}}\left(\mathbb{R}^{d}\right)} .
\end{aligned}
$$

Now by interpolation we obtain for $d=1,2,3,4$ :

$\|u\|_{L^{\frac{4+d}{d}}([t, t+\Delta t]) L^{\frac{8+2 d}{d}}\left(\mathbb{R}^{d}\right)} \leq(\Delta t)^{\frac{d}{d+4}}\|u\|_{L^{\infty}([t, t+\Delta t]) L^{2}\left(\mathbb{R}^{d}\right)}^{\frac{4-d}{d+4}}\|u\|_{L^{\infty}([t, t+\Delta t]) H^{1}\left(\mathbb{R}^{d}\right)}^{\frac{2 d}{d+4}}$, which, according to (1.6), leads to

$$
\left\|u^{\frac{4}{d}} \nabla u\right\|_{L_{t}^{1} L_{x}^{2}} \leq(\Delta t)^{\frac{d}{d+4}}\left\|u_{0}\right\|_{L^{2}\left(\mathbb{R}^{d}\right)}^{\frac{4-d}{d+4}}\|u\|_{S^{1}[t, t+\Delta t]}^{\frac{2 d}{d+4}}\|u\|_{S^{1}[t, t+\Delta t]} .
$$


Since by Lemma 4.1 it holds

$\|u\|_{L^{\infty}(] t, t+\Delta t[) H^{1}}+\left\|(\Delta)^{\frac{s+1}{2}} u\right\|_{L^{2}(] t, t+\Delta t[) L_{x}^{2}} \lesssim\|u(t)\|_{H^{1}}+\left\||u|^{\frac{4}{d}} \nabla u\right\|_{L^{1}(] t, t+\Delta t[) L_{x}^{2}}$, we finally get

$\|u\|_{S^{1}[t, t+\Delta t]}+\left\|(-\Delta)^{\frac{s+1}{2}} u\right\|_{L^{2}(] t, t+\Delta t[) L^{2}} \lesssim\|u(t)\|_{H^{1}}+(\Delta t)^{\frac{d}{d+4}}\left\|u_{0}\right\|_{L^{2}\left(\mathbb{R}^{d}\right)}^{\frac{4-d}{d+4}}\|u\|_{S^{1}[t, t+\Delta t]}^{\frac{2 d}{d+4}+1}$.

In view of (2.1) and a continuity argument, it follows that $u \in C([t, t+$ $\left.\Delta t] ; H^{1}\left(\mathbb{R}^{d}\right)\right)$ for some $\Delta t \sim\left\|u_{0}\right\|_{L^{2}\left(\mathbb{R}^{d}\right)}^{\frac{d-4}{d}}\|u(t)\|_{H^{1}\left(\mathbb{R}^{d}\right)}^{-2}$ and

$$
\|u\|_{S^{1}[t, t+\Delta t]}+\left\|(-\Delta)^{\frac{s+1}{2}} u\right\|_{L^{2}(] t, t+\Delta t[) L^{2}} \leq 2\left\|u_{0}\right\|_{H^{1}} .
$$

In the same way

$$
\|u\|_{S^{0}[t, t+\Delta t]} \lesssim\|u(t)\|_{L^{2}\left(\mathbb{R}^{d}\right)}+(\Delta t)^{\frac{d}{d+4}}\left\|u_{0}\right\|_{L^{2}\left(\mathbb{R}^{d}\right)}^{\frac{4-d}{4+d}}\|u\|_{S^{1}[t, t+\Delta t]}^{2 \frac{d}{d+4}}\|u\|_{S^{0}[t, t+\Delta t]}
$$

which ensures that

$$
\|u\|_{S^{0}[t, t+\Delta t]} \leq 2\left\|u_{0}\right\|_{L^{2}\left(\mathbb{R}^{d}\right)} .
$$

Proof of Proposition 4.1 : According to (4.7), each interval $\left[t_{k}, t_{k+1}\right]$, can be divided into $k$ intervals, $\left[\tau_{k}^{j}, \tau_{k}^{j+1}\right]$ of length less that $\lambda\left(t_{k}\right)$. From (3.6), we have

$$
\left.\left|E\left(u\left(\tau_{k}^{j+1}\right)\right)-E\left(u\left(\tau_{k}^{j}\right)\right)\right| \lesssim\left|\int_{\tau_{k}^{j}}^{\tau_{k}^{j+1}} \int_{\mathbb{R}^{d}}(-\Delta)^{s} u\right| u\right|^{\frac{4}{d}} \bar{u} d x \mid .
$$

For notation convenience we set $\Theta=] \tau_{k}^{j}, \tau_{k}^{j+1}\left[\times \mathbb{R}^{d}\right.$. By Cauchy-Schwarz, it holds

$$
\int_{\tau_{k}^{j}}^{\tau_{k}^{j+1}} \int_{\mathbb{R}^{d}}(-\Delta)^{s} u|u|^{\frac{4}{d}} \bar{u} d x d t \leq\left\|(-\Delta)^{\frac{s}{2}}\left(u|u|^{\frac{4}{d}}\right)\right\|_{L^{\frac{4+2 d}{d}}(\Theta)}\left\|(-\Delta)^{\frac{s}{2}} u\right\|_{L^{\frac{4+2 d}{d}}(\Theta)}
$$

and, by interpolation, we have

$$
\left\|(-\Delta)^{\frac{s}{2}} u\right\|_{L^{\frac{4+2 d}{d}}(\Theta)}^{2} \leq\|\nabla u\|_{L^{\frac{4+2 d}{d}}(\Theta)}^{2 s}\|u\|_{L^{\frac{4+2 d}{d}}(\Theta)}^{2-2 s} .
$$

Noticing that the fractional Leibniz rule leads to

$\left\|(-\Delta)^{\frac{s}{2}}\left(|u|^{\frac{4}{d}} u\right)\right\|_{L^{\frac{4+2 d}{4+d}}(\Theta)} \lesssim\left\|(-\Delta)^{\frac{s}{2}} u\right\|_{L^{\frac{4+2 d}{d}}(\Theta)}\left\|u^{\frac{4}{d}}\right\|_{L^{\frac{4+2 d}{4}}(\Theta)} \lesssim\left\|(-\Delta)^{\frac{s}{2}} u\right\|_{L^{\frac{4+2 d}{d}}}\|u\|_{L^{\frac{4+2 d}{d}}(\Theta)}^{\frac{4}{d}}$, we finally obtain

$$
\left|E\left(u\left(\tau_{k}^{j+1}\right)\right)-E\left(u\left(\tau_{k}^{j}\right)\right)\right| \lesssim\|u\|_{L^{\frac{4+2 d}{d}}(\Theta)}^{\frac{4}{d}}\|\nabla u\|_{L^{\frac{4+2 d}{d}}(\Theta)}^{2 s}\|u\|_{L^{\frac{4+2 d}{d}}(\Theta)}^{2-2 s} .
$$

Since $\left(\frac{4}{d}+2, \frac{4}{d}+2\right)$ is an admissible pair, Lemma 4.2 yields

$$
\left|E\left(u\left(\tau_{k}^{j+1}\right)\right)-E\left(u\left(\tau_{k}^{j}\right)\right)\right| \lesssim \lambda\left(t_{k}\right)^{-2 s}
$$

and summing over $j$ we get

$$
\left|E\left(u\left(t_{k+1}\right)\right)-E\left(u\left(t_{k}\right)\right)\right| \lesssim k \lambda\left(t_{k}\right)^{-2 s} .
$$

Finally, taking $T^{+}=T$ and summing from $k_{0}$ to $k^{+}$, we obtain:

$$
\left|E\left(u\left(T^{+}\right)\right)-E\left(u_{0}\right)\right| \lesssim k^{+} \lambda^{-2 s}\left(T^{+}\right) \lesssim \log (\lambda(T)) \lambda^{-2 s}(T) .
$$


Note that the growth of the energie is small with to respect $\frac{1}{\lambda^{2}}$, because $s<1$.

Let us now proceed with the momentum. According to (1.8) we have :

But

$$
\left|P\left(u\left(\tau_{k}^{j+1}\right)\right)-P\left(u\left(\tau_{k}^{j}\right)\right)\right| \lesssim \int_{\tau_{k}^{j}}^{\tau_{k}^{j+1}}\left|\int_{\mathbb{R}^{d}} \overline{\nabla u}(-\Delta)^{s} u\right| .
$$

$\left|\int_{\mathbb{R}^{d}} \overline{\nabla u}(-\Delta)^{s} u\right|=\left|\int_{\mathbb{R}^{d}}(-\Delta)^{\frac{s}{2}+\frac{1}{4}} u(-\Delta)^{\frac{s}{2}-\frac{1}{4}} \overline{\nabla u}\right| \leq\left\|(-\Delta)^{\frac{s}{2}+\frac{1}{4}} u\right\|_{L^{2}\left(\mathbb{R}^{d}\right)}\left\|(-\Delta)^{\frac{s}{2}-\frac{1}{4}} \nabla u\right\|_{L^{2}\left(\mathbb{R}^{d}\right)}$

with

and, by interpolation,

$$
\left\|(-\Delta)^{\frac{s}{2}-\frac{1}{4}} \nabla u\right\|_{L^{2}\left(\mathbb{R}^{d}\right)}=\left\|(-\Delta)^{\frac{s}{2}+\frac{1}{4}} u\right\|_{L^{2}\left(\mathbb{R}^{d}\right)}
$$

$$
\left\|(-\Delta)^{\frac{s}{2}+\frac{1}{4}} u\right\|_{L^{2}\left(\mathbb{R}^{d}\right)}^{2} \leq\left\|(-\Delta)^{\frac{s}{2}+\frac{1}{2}} u\right\|_{L^{2}\left(\mathbb{R}^{d}\right)}^{2 \theta}\|u\|_{L^{2}\left(\mathbb{R}^{d}\right)}^{2-2 \theta},
$$

where $0<\theta=\frac{2 s+1}{2 s+2}<1$. Therefore we get

$$
\left|P\left(u\left(\tau_{k}^{j+1}\right)\right)-P\left(u\left(\tau_{k}^{j}\right)\right)\right| \lesssim\left(\tau_{k+1}-\tau_{k}\right)^{1-\theta}\left\|u_{0}\right\|_{L^{2}\left(\mathbb{R}^{d}\right)}^{2-2 \theta}\left\|(-\Delta)^{\frac{s}{2}+\frac{1}{2}} u\right\|_{L^{2}(\Theta)}^{2 \theta}
$$

and Lemma 4.2 ensures that

$$
\left|P\left(u\left(\tau_{k}^{j+1}\right)\right)-P\left(u\left(\tau_{k}^{j}\right)\right)\right| \lesssim \lambda^{2-2 \theta} \lambda^{-2 \theta}=\lambda^{2-4 \theta}=\lambda^{\frac{-2 s}{s+1}} .
$$

Summing over $j$ we obtain that:

$$
\mid P\left(u\left(\tau_{k}\right)-P\left(u\left(\tau_{k}\right)\right) \mid \lesssim k \lambda^{\frac{-2 s}{s+1}}\right.
$$

and summing from $k_{0}$ to $k^{+}$, we finally get

$$
\left.\mid P\left(u\left(T^{+}\right)\right)-P\left(u_{0}\right)\right) \mid \lesssim \log (\lambda(T)) \lambda(T)^{\frac{-2 s}{s+1}} .
$$

Note that the growth of the momentum is small with respect $\frac{1}{\lambda}$ since $1-$ $\frac{2 s}{s+1}>0$.

\section{REFERENCES}

[1] P. Antonelli and C. Sparber. Global well-posedness for cubic NLS with nonlinear damping. Comm. Partial Differential Equations, 35 (2010) 48324845.

[2] H. Berestycki and P.-L. Lions. Nonlinear scalar field equations. II. Existence of infinitely many solutions. Arch. Rational Mech. Anal., 82(1983):347-375.

[3] T. Cazenave. Semilinear Schrödinger equations, volume 10 of Courant Lecture Notes in Mathematics. New York University Courant Institute of Mathematical Sciences, New York, 2003.

[4] T. Cazenave and F. Weissler. The Cauchy problem for the critical nonlinear Schrdinger equation. Nonlinear Anal. 14 (1990), 807-836.

[5] J. Colliander and P. Raphael. Rough blowup solutions to the $L^{2}$ critical NLS. Math. Ann., 345(2009):307-366.

[6] M. Darwich. Blowup for the Damped $L^{2}$ critical nonlinear Shrödinger equations. Advances in Differential Equations. volume 17, Numbers 3-4 (2012),337-367.

[7] B. Dodson. Global well-posedness and scattering for the mass critical nonlinear Schrdinger equation with mass below the mass of the ground state. Advances in Mathematics 285 (2015), 1589-1618.

[8] G.Fibich. Self-focusing in the damped nonlinear Schrdinger equation. SIAM J. Appl. Math, 61 (2001), no. 5, 16801705.

[9] G. Fibich and F. Merle. Self-focusing on bounded domains. Phys. D, 155(2001):132158. 
[10] G. Fibich and M. Klein. Nonlinear-damping continuation of the nonlinear Schrdinger equation-a numerical study. Physica D, 241 (2012), 519-527.

[11] A. Friedman Partial Differential Equations.

[12] T. Hmidi and S. Keraani. Blowup theory for the critical nonlinear Schrödinger equations revisited. Int. Math. Res. Not., 46(2005):2815-2828.

[13] T. Kato. On nonlinear Schrödinger equations Ann. Inst. H. Poincaré Phys. Théor., 46(1987):113-129.

[14] M.K Kwong. Uniqueness of positive solutions of $\Delta u-u+u^{p}=0$ in $\mathbf{R}^{n}$. Arch. Rational Mech. Anal., 105(1989):243-266.

[15] P.-L. Lions. The concentration-compactness principle in the calculus of variations. The locally compact case. II. Ann. Inst. H. Poincaré Anal. Non Linéaire, 1(1984):223283.

[16] F. Merle. Determination of blow-up solutions with minimal mass for nonlinear Schr öinger equations with critical power, Duke Math. J. 69:2, (1993), 427-454.

[17] F. Merle and P. Raphael. Blow up dynamic and upper bound on the blow up rate for critical nonlinear Schrödinger equation. In Journées "Équations aux Dérivées Partielles" (Forges-les-Eaux, 2002), pages Exp. No. XII, 5. Univ. Nantes, Nantes, 2002.

[18] F. Merle and P. Raphael. Sharp upper bound on the blow-up rate for the critical nonlinear Schrödinger equation. Geom. Funct. Anal., 13(2003):591-642.

[19] F. Merle and P. Raphael. On universality of blow-up profile for $L^{2}$ critical nonlinear Schrödinger equation. Invent. Math., 156(2004):565-672.

[20] F. Merle and P. Raphael. Profiles and quantization of the blow up mass for critical nonlinear Schrödinger equation. Comm. Math. Phys., 253(2005):675-704.

[21] F. Merle and P. Raphael. On a sharp lower bound on the blow-up rate for the $L^{2}$ critical nonlinear Schrödinger equation. J. Amer. Math. Soc., 19(2006):37-90 (electronic).

[22] C. Miao, B. Yuan and B. Zhang Well-posedness of the Cauchy problem for the fractional power dissipative equations.Nonlinear Analysis, 68 (2008) 461-484

[23] E. Ott and R.N. Sudan.Damping of Solitary Waves. Phys. Fluids, 13 (1970) , 14321434.

[24] M. Ohta and G. Todorova. Remarks on global existence and blowup for damped nonlinear Schrödinger equations. Discrete Contin. Dyn. Syst., 23(2009):1313-1325.

[25] T. Passot, C. Sulem and P.L. Sulem. Linear versus nonlinear dissipation for critical NLS equation. Physica D, 203 (2005) 167184

[26] F. Planchon and P. Raphaël. Existence and stability of the log-log blow-up dynamics for the $L^{2}$-critical nonlinear Schrödinger equation in a domain. Ann. Henri Poincaré, 8(2007):1177-1219.

[27] P. Raphael. Stability of the log-log bound for blow up solutions to the critical non linear Schrödinger equation. Math. Ann., 331(2005):577-609.

[28] M. Tsutsumi. Nonexistence of global solutions to the Cauchy problem for the damped nonlinear Schrödinger equations. SIAM J. Math. Anal., 15(1984):357-366.

[29] M.I. Weinstein. Nonlinear Schrödinger equations and sharp interpolation estimates. Comm. Math. Phys., 87(1982/83):567-576.

Mohamad Darwich: Faculté des sciences de l'Université Libanaise, Département DE MAThÉmatiques PuREs, Hadath, Liban

E-mail address: Mohamad.Darwich@lmpt.univ-tours.fr

Luc Molinet: Laboratoire de Mathématiques et Physique Théorique, Universté Francois Rabelais Tours, CNRS UMR 7350- FÉdération Denis Poisson, Parc Grandmont, 37200 Tours, France

E-mail address: Luc.Molinet@lmpt.univ-tours.fr 